\title{
EPIMORPHISMS, DEFINABILITY AND CARDINALITIES
}

\author{
T. MORASCHINI, J.G. RAFTERY, AND J.J. WANNENBURG
}

\begin{abstract}
We characterize, in syntactic terms, the ranges of epimorphisms in an arbitrary class of similar first-order structures (as opposed to an elementary class). This allows us to strengthen a result of Bacsich, as follows: in any prevariety having at most $\mathfrak{s}$ non-logical symbols and an axiomatization requiring at most $\mathfrak{m}$ variables, if the epimorphisms into structures with at most $\mathfrak{m}+\mathfrak{s}+\aleph_{0}$ elements are surjective, then so are all of the epimorphisms. Using these facts, we formulate and prove manageable 'bridge theorems', matching the surjectivity of all epimorphisms in the algebraic counterpart of a logic $\vdash$ with suitable infinitary definability properties of $\vdash$, while not making the standard but awkward assumption that $\vdash$ comes furnished with a proper class of variables.
\end{abstract}

\section{INTRODUCTION}

'Bridge theorems' of abstract algebraic logic [8, 14, 16, 17] have the form

$\vdash$ has logical property $P$ iff $\mathrm{K}$ has algebraic property $Q$,

where $\vdash$ is an algebraizable logic and $\mathrm{K}$ is its algebraic counterpart - in which case $\mathrm{K}$ is a prevariety, at least. Examples include connections between Beth definability properties and the surjectivity of epimorphisms. Roughly speaking, Beth properties ask that, whenever a set $\Gamma$ of formal assertions about $\vec{x}, \vec{z}$ defines $\vec{z}$ implicitly in terms of $\vec{x}$, then it does so explicitly as well. (Greater precision will be offered in Section 7,)

In fact, there are bridge theorems characterizing several different Bethstyle properties by demands that various kinds of epimorphism be surjective. The first of these was proved by Németi; see [24, Thm. 5.6.10]. Others were

Key words and phrases. Epimorphism, prevariety, quasivariety, Beth definability, algebraizable logic, equivalential logic.

2010 Mathematics Subject Classification: 03G27, 08C15.

This work received funding from the European Union's Horizon 2020 research and innovation programme under the Marie Sklodowska-Curie grant agreement No 689176 (project "Syntax Meets Semantics: Methods, Interactions, and Connections in Substructural logics"). The first author was also supported by the project GA17-04630S of the Czech Science Foundation (GAČR). The second author was supported in part by the National Research Foundation of South Africa (UID 85407). The third author was supported by the DST-NRF Centre of Excellence in Mathematical and Statistical Sciences (CoE-MaSS), South Africa. Opinions expressed and conclusions arrived at are those of the authors and are not necessarily to be attributed to the CoE-MaSS. 
provided in [26, 27, 6, 11]. Concrete antecedents involving special families of logics can be found in Maksimova's work, e.g., see [19, 31, 32].

Blok and Hoogland [6] showed that the straightforward ES propertyi.e., the demand that all epimorphisms in $\mathrm{K}$ be surjective corresponds to an infinite version of the Beth property, where no cardinal is assumed to bound the lengths of the sequences $\vec{x}, \vec{z}$, nor the size of $\Gamma$. When testing for implicit definability, we need to substitute expressions for the variables $\vec{z}$, and this may introduce fresh variables. For such reasons, in the general bridge theorem connecting the ES and infinite Beth properties, the logic $\vdash$ needs to be formulated with a proper class of variables.

On the other hand, many familiar algebraizable logics are finitary, with only countably many connectives, and are formalized using a countable set of variables - as nothing more is required for their axiomatization. When working with such logics, one would prefer a version of the infinite Beth property that also presupposes only a set of variables, but is still provably equivalent to the unrestricted ES property for the algebraic counterpart.

It seems, however, that the published literature of abstract algebraic logic contains no such bridge theorem. Analyzing the core proof in [6], we find that the gap would be filled by the following claim, where $\mathfrak{m}, \mathfrak{s}$ are cardinals:

If a prevariety $\mathrm{K}$ lacking the ES property has just $\mathfrak{s}$ operation symbols and an axiomatization that uses only $\mathfrak{m}$ variables, then there is a non-surjective K-epimorphism $\boldsymbol{A} \rightarrow \boldsymbol{B}$, where $\boldsymbol{B}$ has at most $\mathfrak{m}+\mathfrak{s}+\aleph_{0}$ elements.

This claim (generalized to first-order structures) is Theorem 5.1 of the present paper. One of its specializations (which follows also from a result of Bacsich [2]) says that

in a quasivariety of countable type, if the epimorphisms into countable structures are surjective, then so are all of the epimorphisms.

We use these facts to obtain more manageable bridge theorems (Theorem 7.6. Corollaries 7.7, 7.8), connecting the unrestricted ES property with suitably localized infinite Beth properties for logics formalized with limited variables and connectives. The bridge theorems cater for all equivalential logics and specialize to the algebraizable ones.

The proof of Theorem 5.1 rests on a general syntactic characterization of the ranges of epimorphisms (Theorem [3.1), which widens the scope of Bacsich [2, Thm. 1] and Campercholi [12, Thm. 3]. (The earlier accounts apply only to classes closed under ultraproducts.)

\section{Atomic Consequence}

We work in the conservative extension NBG of ZFC (i.e., in the class theory of von Neumann, Bernays and Gödel, including the axiom of choice). 
The cardinality of the signature of a first-order language is the sum of the cardinalities of its (disjoint) sets of operation and relation symbols. The ranks of these symbols are assumed finite, and nonzero in the case of relations. Only first-order signatures will be considered. A signature is algebraic if it has no relation symbols.

For a given signature, a structure denoted by $\mathcal{A}$ is assumed to have universe $A$ (a non-empty set) and algebra reduct $\boldsymbol{A}=\langle A ; O\rangle$ for a suitable set $O$ of operations on $A$, so that $\mathcal{A}=\langle A ; O, R\rangle$ for a suitable set $R$ of relations on $A$. The subalgebra of $\boldsymbol{A}$ and the substructure of $\mathcal{A}$ generated by a set $X \subseteq A$ have the same universe; the latter is denoted by $\operatorname{Sg}^{\mathcal{A}}(X)$. A homomorphism $h: \mathcal{A} \rightarrow \mathcal{B}$ is understood to preserve the relations in $R$ (as well as the operations in $O$ ), but it need not reflect the relations. As usual, the kernel $\left\{\left\langle a, a^{\prime}\right\rangle \in A^{2}: h(a)=h\left(a^{\prime}\right)\right\}$ of $h$ is denoted by ker $h$.

It is convenient here to have recourse to a fixed proper class Var of variables. For each subset $X$ of $\operatorname{Var}$, we use $\boldsymbol{T}(X)$ to denote the absolutely free algebra (a.k.a. the term algebra) generated by $X$, with respect to the operation symbols of the signature under discussion. Given a class $\Sigma \cup\{p\}$ of expressions over Var, the set of variables occurring in $p$ shall be denoted by $\operatorname{var}(p)$, while $\operatorname{var}(\Sigma):=\bigcup_{s \in \Sigma} \operatorname{var}(s)$.

Recall that the atomic formulas of a signature are either equations $\varphi \approx \psi$ or expressions $r\left(\varphi_{1}, \ldots, \varphi_{n}\right)$, where $r$ is a relation symbol and $\varphi, \psi, \varphi_{1}, \ldots, \varphi_{n}$ are terms over Var. We tacitly identify $\varphi \approx \psi$ with the pair $\langle\varphi, \psi\rangle$.

If, upon introducing a set $\Sigma$ of atomic formulas, we denote it as $\Sigma(\vec{x})$, this signifies that the elements of $\operatorname{var}(\Sigma)$ all belong to the sequence $\vec{x}$ of distinct variables. (Strictly speaking, like any sequence, $\vec{x}$ is a function whose domain is some ordinal $\delta$, so $|\vec{x}|=|\delta|$, which need not be finite.) In this context, given a structure $\mathcal{A}$ of matching signature, the notation $\mathcal{A} \models \Sigma(\vec{a})$ has the standard model-theoretic meaning, which entails in particular that $\vec{a}$ is a $\delta$ indexed sequence of not necessarily distinct elements of $A$ (briefly: $\vec{a} \in A$ ).

Definition 2.1. For any class $\mathrm{K}$ of similar structures, we use the notation $\Sigma(\vec{x}) \models_{\mathrm{K}} p(\vec{x})$ (or $\left.\Sigma \models_{\mathrm{K}} p\right)$ to signify that $\Sigma(\vec{x}) \cup\{p(\vec{x})\}$ is a set of atomic formulas in the signature of $\mathrm{K}$ and

whenever $\mathcal{A} \in \mathrm{K}$ and $\mathcal{A} \models \Sigma(\vec{a})$ (where $\vec{a} \in A$ ), then $\mathcal{A} \models p(\vec{a})$.

The displayed demand may be paraphrased a little more precisely as

for any $\mathcal{A} \in \mathrm{K}$ and any homomorphism $h: \boldsymbol{T}(\operatorname{var}(\Sigma \cup\{p\})) \rightarrow \boldsymbol{A}$, if $\mathcal{A} \models \Sigma(h[\vec{x}])$, then $\mathcal{A} \models p(h[\vec{x}])$.

Here, $h[\vec{x}]$ is the sequence obtained by applying $h$ to every item of $\vec{x}$. When $\Sigma \models_{\mathrm{K}} p$, we say that ' $\Sigma / p$ is validated by (each member of) $\mathrm{K}$ '. The ordered pair $\Sigma / p$ will then be referred to as an (atomic) implication; we call it a quasi-atomic formula if $\Sigma$ is finite.1

\footnotetext{
${ }^{1}$ It could be rendered more suggestively as $(\& \Sigma) \Longrightarrow p$, or as $\left(\&_{s \in \Sigma} s\right) \Longrightarrow p$.
} 
In this paper, we shall not need to deal with formulas more complex than implications. Any syntactic substitution instance $\Sigma(\vec{\varphi})$ of $\Sigma(\vec{x})$ shall have the form $\{s(h[\vec{x}]): s \in \Sigma\}$, where $h: \boldsymbol{T}(\operatorname{var}(\Sigma)) \rightarrow \boldsymbol{T}(Y)$ is a homomorphism, $Y$ being a subset of $\operatorname{Var}$.

The class operator symbols $\mathbb{I}, \mathbb{H}, \mathbb{S}, \mathbb{P}$ and $\mathbb{P}_{\mathbb{U}}$ stand, respectively, for closure under isomorphic and homomorphic (surjective) images, substructures, (set-indexed) direct products and ultraproducts.

A class $\mathrm{K}$ of similar structures is said to be axiomatized by a class $\Xi$ of implications if $\mathrm{K}$ is the class of all structures validating all of the implications in $\Xi$. In this case, $K$ is a prevariety, i.e., it is closed under $\mathbb{I}, \mathbb{S}$ and $\mathbb{P}$.

Conversely, every prevariety is axiomatized by a class $\Xi$ of implications; see Banaschewski and Herrlich [3]. The claim that we cannot always find a set to play the role of $\Xi$ (or equivalently, of $\operatorname{var}(\Xi)$ ) is consistent with NBG. Its negation (i.e., the claim that sets suffice) is consistent with NBG if huge cardinals exist. These facts were established by Adámek [1].

A prevariety can be axiomatized by a class - w.l.o.g. a set - of quasiatomic [resp. atomic] formulas iff it is closed under $\mathbb{P}_{\mathbb{U}}[$ resp. $\mathbb{H}]$, in which case we call it a quasivariety [resp. a variety], even if its members are not pure algebras. The reader may consult [20, Ch. 2] for proofs of these well-known results (which originate in [5, 21, 33]).

Definition 2.2. For any class $K$ of similar structures and any infinite cardinal $\mathfrak{m}$, the relation $\models_{\mathrm{K}}$ is said to be $\mathfrak{m}$-compact provided that,

$$
\text { whenever } \Sigma \models_{\mathrm{K}} p \text {, then } \Sigma^{\prime} \models_{\mathrm{K}} p \text { for some } \Sigma^{\prime} \subseteq \Sigma \text { with }\left|\Sigma^{\prime}\right|<\mathfrak{m} .
$$

We say that $\models_{\mathrm{K}}$ is finitary if it is $\aleph_{0}$-compact.

If $\mathrm{K}$ is closed under $\mathbb{P}_{\mathbb{U}}$ (e.g., if $\mathrm{K}$ is a quasivariety), then $\models_{K}$ is finitary. The terminology of Definition 2.2 applies, more generally, to arbitrary relations from subsets of a class to elements of the same class.

\section{EPIMORPHISMS}

A morphism $h$ in a category $\mathrm{C}$ is called a $(\mathrm{C}-)$ epimorphism provided that, for any two C-morphisms $f, g$ from the co-domain of $h$ to a single object,

$$
\text { if } f \circ h=g \circ h \text {, then } f=g \text {. }
$$

We shall not distinguish notationally between a class $\mathrm{K}$ of similar (firstorder) structures and the concrete category of all homomorphisms between its members. Clearly, within such a category, every surjective homomorphism is an epimorphism. If the converse holds, then $\mathrm{K}$ is said to have the epimorphism surjectivity property, or briefly, the ES property.

A substructure $\mathcal{D}$ of a structure $\mathcal{B} \in \mathrm{K}$ is said to be $(\mathrm{K}-)$ epic in $\mathcal{B}$ if each homomorphism from $\mathcal{B}$ to a member of $\mathrm{K}$ is determined by its restriction to $\mathcal{D}$. (This is equivalent to the demand that the inclusion map $\mathcal{D} \rightarrow \mathcal{B}$ be an epimorphism, provided that $\mathrm{K}$ is closed under $\mathbb{S}$.) Of course, a $\mathrm{K}$ morphism $h: \mathcal{A} \rightarrow \mathcal{B}$ is an epimorphism iff $h[\mathcal{A}]$ is an epic substructure of 
$\mathcal{B}$. Therefore, $\mathrm{K}$ has the ES property iff each of its members has no proper epic substructure.

The significance of epimorphisms for formal deductive systems (alluded to in the introduction) will be elaborated in Section 7 . For the moment, however, structures are our concern. Already in the context of algebras, we may recall that rings and distributive lattices each form varieties lacking the ES property. This reflects the absence of unary terms defining multiplicative inverses in rings, and complements in distributive lattices, despite the uniqueness of those entities when they exist.

The connection between such 'implicitly defined' constructs and epimorphisms was remarked upon in the algebraic literature long ago (e.g., see Freyd [18, p. 93] and Isbell [28]). It was given a syntactically sharper characterization by Bacsich [2, Thm. 1] and by Campercholi [12, Thm. 3] (see Remark 3.3 below), but their accounts are confined, respectively, to universal classes and to classes closed under ultraproducts. With logical applications in mind, we extend the result to arbitrary classes in the next theorem.

Theorem 3.1. Let $\mathrm{K}$ be any class of similar structures, $\mathcal{A}$ a substructure of $\mathcal{B} \in \mathrm{K}$ and $Z \subseteq B \backslash A$, where $\mathcal{B}=\operatorname{Sg}^{\mathcal{B}}(A \cup Z)$. Then the following conditions are equivalent.

(i) $\mathcal{A}$ is $\mathrm{K}$-epic in $\mathcal{B}$.

(ii) For each $b \in Z$, there is a set $\Sigma=\Sigma(\vec{x}, \vec{z}, v)$ of atomic formulas such that $\mathcal{B} \models \Sigma(\vec{a}, \vec{c}, b)$ for suitable $\vec{a} \in A$ and $\vec{c} \in B$, and

$$
\Sigma\left(\vec{x}, \vec{z}, v_{1}\right) \cup \Sigma\left(\vec{x}, \vec{y}, v_{2}\right) \models_{\mathrm{K}} v_{1} \approx v_{2} .
$$

In this case, for each $b \in Z$, we can arrange that $|\vec{z}| \leq|Z|$ and $\vec{c} \in Z$.

Proof. (ii) $\Rightarrow$ (iii): Let $\vec{x}_{A}$ and $\vec{x}_{Z}$ be sequences of variables whose disjoint ranges, $\left\{x_{a}: a \in A\right\}$ and $\left\{x_{b}: b \in Z\right\}$, are bijective copies of $A$ and $Z$, respectively. Let $\boldsymbol{T}=\boldsymbol{T}\left(\vec{x}_{A}, \vec{x}_{Z}\right)$ be the absolutely free algebra generated by the combined ranges of $\vec{x}_{A}$ and $\vec{x}_{Z}$. Let $h: \boldsymbol{T} \rightarrow \boldsymbol{B}$ be the homomorphism such that $h\left(x_{a}\right)=a$ and $h\left(x_{b}\right)=b$ for all $a \in A$ and $b \in Z$. Note that $h$ is surjective, as $A \cup Z$ generates $\boldsymbol{B}$.

Now fix $b \in Z$. Let $\vec{x}_{Z \backslash\{b\}}$ denote the subsequence of $\vec{x}_{Z}$ whose range omits $x_{b}$. For notational convenience, assume that $\vec{x}_{Z}$ is ordered as $\vec{x}_{Z \backslash\{b\}}, x_{b}$.

Let $\Sigma=\Sigma\left(\vec{x}_{A}, \vec{x}_{Z \backslash\{b\}}, x_{b}\right)$ be the set of all atomic formulas $r\left(\vec{x}_{A}, \vec{x}_{Z}\right)$ such that $\mathcal{B} \models r\left(h\left[\vec{x}_{A}\right], h\left[\vec{x}_{Z}\right]\right)$. Note that ker $h \subseteq \Sigma$, as $\Sigma$ includes equations.

Because $\mathcal{B} \models \Sigma\left(h\left[\vec{x}_{A}\right], h\left[\vec{x}_{Z \backslash\{b\}}\right], h\left(x_{b}\right)\right)$, where $h\left(x_{b}\right)=b$ and the items in $h\left[\vec{x}_{A}\right]$ and $h\left[x_{Z \backslash\{b\}}\right]$ belong to $A$ and $Z$ (respectively), it remains only to prove that $\Sigma\left(\vec{x}_{A}, \vec{x}_{Z \backslash\{b\}}, x_{b}\right) \cup \Sigma\left(\vec{x}_{A}, \vec{y}_{Z \backslash\{b\}}, y_{b}\right) \models_{\mathrm{K}} x_{b} \approx y_{b}$.

Let $\mathcal{C} \in \mathrm{K}$ and let $g_{1}, g_{2}: \boldsymbol{T} \rightarrow \boldsymbol{C}$ be homomorphisms that agree on $\vec{x}_{A}$, where $\mathcal{C} \models r\left(g_{1}\left[\vec{x}_{A}\right], g_{1}\left[\vec{x}_{Z}\right]\right)$ and $\mathcal{C} \models r\left(g_{2}\left[\vec{x}_{A}\right], g_{2}\left[\vec{x}_{Z}\right]\right)$ for all $r\left(\vec{x}_{A}, \vec{x}_{Z}\right) \in \Sigma$. Then ker $h \subseteq \operatorname{ker} g_{1} \cap \operatorname{ker} g_{2}$. We must show that $g_{1}\left(x_{b}\right)=g_{2}\left(x_{b}\right)$.

Let $j \in\{1,2\}$. Because $h$ is surjective and $\operatorname{ker} h \subseteq \operatorname{ker} g_{j}$, the function $h(\varphi) \mapsto g_{j}(\varphi)$ is a well defined homomorphism $f_{j}: \boldsymbol{B} \rightarrow \boldsymbol{C}$. In fact, $f_{j}$ 
is a homomorphism from $\mathcal{B}$ to $\mathcal{C}$, by the definitions of $\Sigma$ and $g_{j}$. For each $a \in A$, we have $f_{j}(a)=f_{j}\left(h\left(x_{a}\right)\right)=g_{j}\left(x_{a}\right)$, but $g_{1}$ and $g_{2}$ agree at $x_{a}$, so $\left.f_{1}\right|_{A}=\left.f_{2}\right|_{A}$. Then $f_{1}=f_{2}$, since $\mathcal{C} \in \mathrm{K}$ and $\mathcal{A}$ is $\mathrm{K}$-epic in $\mathcal{B}$. Therefore, $g_{1}\left(x_{b}\right)=f_{1}(b)=f_{2}(b)=g_{2}\left(x_{b}\right)$, as required.

(iii) $\Rightarrow$ (ii): Let $g, h: \mathcal{B} \rightarrow \mathcal{C} \in \mathrm{K}$ be homomorphisms, with $\left.g\right|_{A}=\left.h\right|_{A}$. We must show that $g=h$. As $A \cup Z$ generates $\mathcal{B}$, it suffices to prove that $\left.g\right|_{Z}=\left.h\right|_{Z}$. Let $b \in Z$, and let $\Sigma$ and $\vec{a} \in A$ and $\vec{c} \in B$ be as in (iii). From $\mathcal{B} \models \Sigma(\vec{a}, \vec{c}, b)$ we infer $\mathcal{C} \models \Sigma(g[\vec{a}], g[\vec{c}], g(b))$ and $\mathcal{C} \models \Sigma(h[\vec{a}], h[\vec{c}], h(b))$. But $g[\vec{a}]=h[\vec{a}]$, as $\left.g\right|_{A}=\left.h\right|_{A}$, so $g(b)=h(b)$, by (1). Thus, $\left.g\right|_{Z}=\left.h\right|_{Z}$.

Remark 3.2. In Theorem 3.1(iii), if $\models_{\mathrm{K}}$ is finitary (e.g., if $\mathrm{K}$ is closed under ultraproducts), then each $\Sigma$ can be chosen finite.

Remark 3.3. Given $\mathcal{A} \in \mathbb{S}(\mathcal{B})$, it is sometimes convenient to define the $\mathrm{K}$-dominion $\operatorname{dom}_{\mathcal{B}}^{\mathrm{K}} \mathcal{A}$ (of $\mathcal{A}$ in $\mathcal{B}$ ) as the set of all $b \in B$ such that any two homomorphisms from $\mathcal{B}$ to a member of $\mathrm{K}$ will agree at $b$ if they agree on $A$. Then $\mathcal{A}$ is $\mathrm{K}$-epic in $\mathcal{B}$ iff $\operatorname{dom}_{\mathcal{B}}^{\mathrm{K}} \mathcal{A}=B$. In the above proof, if we choose $Z=B \backslash A$, then the argument shows that, for any $b \in B$, we have $b \in \operatorname{dom}_{\mathcal{B}}^{\mathrm{K}} \mathcal{A}$ iff there exist a set of atomic formulas $\Sigma(\vec{x}, \vec{z}, v)$ such that $\mathcal{B} \models \Sigma(\vec{a}, \vec{c}, b)$ for suitable $\vec{a} \in A$ and $\vec{c} \in B$, and (1) holds. Restricting to the case where $\mathrm{K}$ is closed under $\mathbb{P}_{\mathbb{U}}$ (whence each $\Sigma$ can be chosen finite, by Remark 3.2 ), we obtain a more elementary proof of the aforementioned result of Bacsich [2, Thm. 1], and likewise Campercholi [12, Thm. 3].2

Remark 3.4. In a structure $\mathcal{B}$, a substructure $\mathcal{A}$ is said to be almost total if $\mathcal{B}=\operatorname{Sg}^{\mathcal{B}}(A \cup Z)$ for some finite $Z \subseteq B$. By Theorem 3.1, the demand that an almost total substructure of $\mathcal{B}$ be $\mathrm{K}$-epic is characterized by the existence of finitely many suitable implications (of possibly infinite length), each having only finitely many variables in the role of $\vec{z}$. We say that $K$ has the weak $E S$ property if no $\mathcal{B} \in \mathrm{K}$ has a proper $\mathrm{K}$-epic almost total substructure. It is pointed out in [6, p. 76] that the meaning of this demand would not change if, in the definition of 'almost total', we required $|Z|=1$.

\section{4. $\mathfrak{m}$-PREVARIETIES}

From now on, $\mathfrak{m}$ shall denote a fixed but arbitrary infinite cardinal. As usual, $\mathfrak{m}^{+}$stands for the cardinal successor of $\mathfrak{m}$.

As was mentioned in Section 2 , our ability to axiomatize arbitrary prevarieties using only sets of variables depends on the set theory in which we work [1]. This justifies our interest in the following classes.

Definition 4.1. An $\mathfrak{m}$-prevariety is a class of structures axiomatized by implications, each of which is formulated in at most $\mathfrak{m}$ variables.

Suppose $\Xi$ is a set of implications axiomatizing $\mathrm{K}$, where $\operatorname{var}(\Xi) \subseteq Y$ and $|Y| \leq \mathfrak{m}$. Then the set of atomic formulas over $Y$ has cardinality at most $\mathfrak{n}:=\mathfrak{m}+\mathfrak{s}$, where $\mathfrak{s}$ is the cardinality of the signature. Therefore, each of the

\footnotetext{
${ }^{2}$ Dominions were introduced (for algebras) by Isbell [28]; also see [10, 11, 25, 37].
} 
implications in $\Xi$ has at most $\mathfrak{n}$ atomic subformulas. From this it follows easily that $\mathrm{K}$ is closed under $\mathfrak{n}^{+}$-reduced products, i.e., for any subfamily $\left\{\mathcal{A}_{i}: i \in I\right\}$ of $\mathrm{K}$ and any $\mathfrak{n}^{+}$-complete filter $D$ over $I$, the reduced product $\prod_{i \in I} \mathcal{A}_{i} / D$ belongs to $\mathrm{K}$. The demand that $D$ be $\mathfrak{n}^{+}$-complete means that, whenever $E \subseteq D$ and $|E| \leq \mathfrak{n}$, then $\bigcap E \in D$. In summary:

Lemma 4.2. (cf. [20, Prop. 2.3.19]) Each $\mathfrak{m}$-prevariety is closed under $(\mathfrak{m}+\mathfrak{s})^{+}$-reduced products, where $\mathfrak{s}$ is the cardinality of the signature.

Theorem 4.3. Let $\mathrm{K}$ be an $\mathfrak{m}$-prevariety, whose signature has cardinality s. Then $\models_{\mathrm{K}}$ is $(\mathfrak{m}+\mathfrak{s})^{+}$- compact.

Proof. Let $\mathfrak{n}=\mathfrak{m}+\mathfrak{s}$ and suppose $\Sigma(\vec{x}) \models_{\mathrm{K}} p(\vec{x})$, where $\vec{x}=x_{0}, x_{1}, \ldots$ is a sequence of (possibly more than $\mathfrak{n}$ ) variables. Let $I=\{\Lambda \subseteq \Sigma:|\Lambda| \leq \mathfrak{n}\}$. For each $\Lambda \in I$, let $I_{\Lambda}=\{\Gamma \in I: \Lambda \subseteq \Gamma\}$, so $I_{\Lambda} \neq \emptyset$ (as $\left.\Lambda \in I_{\Lambda}\right)$. Define

$$
D=\left\{J \subseteq I: J \supseteq I_{\Lambda} \text { for some } \Lambda \in I\right\},
$$

so $\emptyset \notin D$, and $D$ is upward closed in the power set of $I$. To see that $D$ is an $\mathfrak{n}^{+}$-complete filter over $I$, let $E \subseteq D$, with $|E| \leq \mathfrak{n}$. For each $J \in E$, choose $\Lambda_{J} \in I$ such that $J \supseteq I_{\Lambda_{J}}$. Let $\Lambda=\bigcup_{J \in E} \Lambda_{J}$. Because $\left|\Lambda_{J}\right| \leq \mathfrak{n}$ for all $J \in E$, we have $|\Lambda| \leq \mathfrak{n} \cdot|E|=\mathfrak{n}$, so $\Lambda \in I$. Also, $\bigcap E \supseteq \bigcap_{J \in E} I_{\Lambda_{J}}=I_{\Lambda}$, so $\bigcap E \in D$, as required.

Assume, with a view to contradiction, that for each $\Lambda \in I$, there exists $\mathcal{A}_{\Lambda} \in \mathrm{K}$ such that $\Lambda \forall_{\left\{\mathcal{A}_{\Lambda}\right\}} p$, i.e., there exists $\vec{a}_{\Lambda}=a_{0}^{\Lambda}, a_{1}^{\Lambda}, \ldots \in A_{\Lambda}$ such that $\mathcal{A}_{\Lambda} \models \Lambda\left(\vec{a}_{\Lambda}\right)$ but $\mathcal{A}_{\Lambda} \not \models p\left(\vec{a}_{\Lambda}\right)$. Let $\mathcal{B}=\prod_{\Lambda \in I} \mathcal{A}_{\Lambda}$ and $\mathcal{A}=\mathcal{B} / D$, so $\mathcal{A} \in \mathrm{K}$, by Lemma 4.2. In particular, $\Sigma \models_{\{\mathcal{A}\}} p$.

Define $\vec{b}=b_{0}, b_{1}, \ldots \in B$ by $b_{k}(\Lambda)=a_{k}^{\Lambda}$, for each $k, \Lambda$. If $s \in \Sigma$, then

$$
\{s\} \in I \text { and } I_{\{s\}} \subseteq \llbracket s(\vec{b}) \rrbracket:=\left\{\Lambda \in I: \mathcal{A}_{\Lambda} \models s\left(b_{0}(\Lambda), b_{1}(\Lambda), \ldots\right)\right\},
$$

so $\llbracket s(\vec{b}) \rrbracket \in D$. Thus, $\mathcal{A} \models \Sigma(\vec{b})$, but $\llbracket p(\vec{b}) \rrbracket=\emptyset \notin D$, so $\mathcal{A} \not \models p(\vec{b})$. This shows that $\Sigma \nvdash_{\{\mathcal{A}\}} p$, a contradiction, so $\Lambda \models_{\mathrm{K}} p$ for some $\Lambda \in I$.

\section{ES PROPERTIES}

A structure is said to be $\mathfrak{n}$-generated (where $\mathfrak{n}$ is a cardinal) if its algebra reduct has a generating subset with at most $\mathfrak{n}$ elements. 'Finitely generated' means $n$-generated for some $n \in \omega$. (Recall that $\mathfrak{m}$ is infinite.)

Theorem 5.1. Let $\mathrm{K}$ be an $\mathfrak{m}$-prevariety whose signature has cardinality $\mathfrak{s}$. Then $\mathrm{K}$ has the ES property iff no structure in $\mathrm{K}$ of cardinality at most $\mathfrak{m}+\mathfrak{s}$ has a proper $\mathrm{K}$-epic substructure.

Proof. Again, let $\mathfrak{n}=\mathfrak{m}+\mathfrak{s}$. Suppose that $\mathrm{K}$ lacks the ES property, i.e., some $\mathcal{B} \in \mathrm{K}$ has a proper $\mathrm{K}$-epic substructure $\mathcal{A}$. We must show that some $\mathcal{C} \in \mathrm{K}$, with $|C| \leq \mathfrak{n}$, has a proper $\mathrm{K}$-epic substructure.

We shall define, recursively, a denumerable sequence $\mathcal{C}_{0}, \mathcal{C}_{1}, \mathcal{C}_{2}, \ldots$ of substructures of $\mathcal{B}$, where $\mathcal{C}_{i} \in \mathbb{S}\left(\mathcal{C}_{i+1}\right)$ for each $i \in \omega$.

First, pick $b \in B \backslash A$ and $a \in A$, and define $\mathcal{C}_{0}=\operatorname{Sg}^{\mathcal{B}}\{a, b\}$, so $C_{0} \not \subset A$. 
Now assume that $\mathcal{C}_{i} \in \mathbb{S}(\mathcal{B})$ has been defined, where $i \in \omega$, and that $C_{i} \not \subset A$. Choose $c \in C_{i} \backslash A$. As $c \in B \backslash A$, Theorem 3.1 shows that there exist a set of atomic formulas $\Sigma(\vec{x}, \vec{z}, v)$ and elements $\vec{a}_{c} \in A$ and $\vec{d}_{c} \in B \backslash A$, such that $\mathcal{B} \models \Sigma\left(\vec{a}_{c}, \vec{d}_{c}, c\right)$ and $\Sigma\left(\vec{x}, \vec{z}, v_{1}\right) \cup \Sigma\left(\vec{x}, \vec{y}, v_{2}\right) \models_{\mathrm{K}} v_{1} \approx v_{2}$. Moreover, $\models_{\mathrm{K}}$ is $\mathfrak{n}^{+}$-compact, by Theorem 4.3, so we may assume that $|\Sigma| \leq \mathfrak{n}$, and hence that $|\operatorname{var}(\Sigma)| \leq \mathfrak{n}$. Consequently, $\left|\vec{a}_{c}\right|,\left|\vec{d}_{c}\right| \leq \mathfrak{n}$. Let $W_{i}$ be the union of (the ranges of) all the sequences $\vec{a}_{c}$ and $\vec{d}_{c}$ such that $c \in C_{i} \backslash A$, so

$$
\left|W_{i}\right| \leq \mathfrak{n} \cdot\left|C_{i}\right| \text {. }
$$

Define $\mathcal{C}_{i+1}=\operatorname{Sg}^{\mathcal{B}}\left(C_{i} \cup W_{i}\right)$.

Let $\mathcal{C} \in \mathbb{S}(\mathcal{B})$ be the (directed) union $\bigcup_{i \in \omega} \mathcal{C}_{i}$, so $\mathcal{C} \in \mathrm{K}$. Now $D:=C \cap A$ is not empty, as it includes $a$, so $D$ is the universe of a substructure $\mathcal{D}$ of $\mathcal{B}$. Also, $\mathcal{D}$ is a proper substructure of $\mathcal{C}$, as $b \in C \backslash D$.

To see that $\mathcal{D}$ is $\mathrm{K}$-epic in $\mathcal{C}$, let $c \in C \backslash D$. Then $c \in C_{i} \backslash A$ for some $i \in \omega$. Pick $\Sigma, \vec{a}_{c}$ and $\vec{d}_{c}$ as in the inductive step. Because the substructure $\mathcal{C}_{i+1}$ of $\mathcal{C}$ includes $\vec{a}_{c}, \vec{d}_{c}, c$ and satisfies $\Sigma\left(\vec{a}_{c}, \vec{d}_{c}, c\right)$, the same is true of $\mathcal{C}$. So, noting that $\vec{a}_{c} \in D$, we infer from Theorem 3.1 that $\mathcal{D}$ is $\mathrm{K}$-epic in $\mathcal{C}$.

As $\mathfrak{s}+\aleph_{0} \leq \mathfrak{n}$, the union $\mathcal{C}$ of the family $\left\{\mathcal{C}_{i}: i \in \omega\right\}$ will have at most $\mathfrak{n}$ elements if every $\mathcal{C}_{i}$ is $\mathfrak{n}$-generated, which we verify by induction. Indeed, $\mathcal{C}_{0}$ is 2-generated, and if some $\mathcal{C}_{i}$ is $\mathfrak{n}$-generated, then so is $\mathcal{C}_{i+1}$, by (2).

The corollary below is due to Bacsich 2]. (For varieties of algebras, it follows from an earlier finding of Isbell [28, Cor. 1.3].)

Corollary 5.2. ([2, Thm. 2]) Let $\mathrm{K}$ be a quasivariety with a countable signature. Then $\mathrm{K}$ has the ES property if and only if no countable member of $\mathrm{K}$ has a proper $\mathrm{K}$-epic substructure.

Proof. Since K can be axiomatized by a set of finite implications, it is an $\aleph_{0}$-prevariety, and the result follows from Theorem 5.1 .

Remark 5.3. An $\aleph_{0}$-prevariety need not be a quasivariety, even if it has a variable-free axiomatization: see [1, p. 45]. Nevertheless, in Corollary 5.2, we cannot strengthen 'countable' to 'finitely generated'. Indeed, a locally finite variety $\mathrm{K}$ of Brouwerian algebras and a proper $\mathrm{K}$-epic subalgebra of a denumerable member of $\mathrm{K}$ are exhibited in [4, Sec. 6], but no finitely generated (i.e., finite) member of $\mathrm{K}$ has a proper $\mathrm{K}$-epic subalgebra, because every variety of Brouwerian algebras has the weak ES property (see Remark 3.4, [6, Thm. 3.14] and [29]).

On the other hand, finitely generated structures do suffice, in quasivarieties, to test the weak ES property itself:

Theorem 5.4. A quasivariety $\mathrm{K}$ has the weak ES property iff no finitely generated member of $\mathrm{K}$ has a proper $\mathrm{K}$-epic substructure.

Proof. Suppose that some $\mathcal{B} \in \mathrm{K}$ has a proper $\mathrm{K}$-epic almost total substructure $\mathcal{A}$. So, $\mathcal{B}=\operatorname{Sg}^{\mathcal{B}}(A \cup Z)$ for some finite non-empty set $Z \subseteq B \backslash A$. Let 
$b \in Z$. Because $\mathrm{K}$ is a quasivariety, Remark 3.2 shows that there exist a finite set of atomic formulas $\Sigma_{b}(\vec{x}, \vec{z}, v)$ and elements $\vec{a}_{b} \in A$ and $\overrightarrow{d_{b}} \in Z$ such that $\mathcal{B} \models \Sigma_{b}\left(\vec{a}_{b}, \vec{d}_{b}, b\right)$ and $\Sigma_{b}\left(\vec{x}, \vec{z}, v_{1}\right) \cup \Sigma_{b}\left(\vec{x}, \vec{y}, v_{2}\right) \models_{\mathrm{K}} v_{1} \approx v_{2}$. As $\operatorname{var}\left(\Sigma_{b}\right)$ is finite, the sequences $\vec{a}_{b}$ and $\vec{d}_{b}$ may be chosen finite.

Let $Y$ be the union of (the ranges of) all the sequences $\vec{a}_{b}$ such that $b \in Z$, so $Y$ is finite. Let $\mathcal{A}^{\prime}=\operatorname{Sg}^{\mathcal{A}} Y$ and $\mathcal{B}^{\prime}=\operatorname{Sg}^{\mathcal{B}}(Y \cup Z)$. Then $\mathcal{A}^{\prime}$ is a proper (almost total) substructure of the finitely generated structure $\mathcal{B}^{\prime} \in \mathrm{K}$. For all $b \in Z$, we have $\vec{a}_{b}, \vec{d}_{b}, b \in B^{\prime}$ and $\mathcal{B}^{\prime} \in \mathbb{S}(\mathcal{B})$, so $\mathcal{B}^{\prime} \models \Sigma_{b}\left(\vec{a}_{b}, \vec{d}_{b}, b\right)$. Then, since $\vec{a}_{b} \in A$ for each $b \in Z$, Theorem 3.1 shows that $\mathcal{A}^{\prime}$ is $\mathrm{K}$-epic in $\mathcal{B}^{\prime}$.

\section{Equivalential and Algebraizable Logics}

For a class $C$, we use $\mathcal{P}(C)$ to denote the class of all subsets of $C$.

Definition 6.1. In a given algebraic signature, a deductive system (briefly, a logic) over a set $X$ is a relation $\vdash \subseteq \mathcal{P}(T(X)) \times T(X)$ satisfying the demands below, whenever $\Gamma \cup \Psi \cup\{\varphi\} \subseteq T(X)$ :

(i) $\Gamma \vdash \varphi$ for all $\varphi \in \Gamma$;

(ii) if $\Gamma \vdash \psi$ for all $\psi \in \Psi$, and $\Psi \vdash \varphi$, then $\Gamma \vdash \varphi$;

(iii) if $\Gamma \vdash \varphi$, then $h[\Gamma] \vdash h(\varphi)$ for all endomorphisms $h$ of $\boldsymbol{T}(X)$.

Item (iii) is called substitution-invariance. In this context, operation symbols and elements of $\mathcal{P}(T(X)) \times T(X)$ are usually called 'connectives' and 'rules', respectively. 3

As the set of logics over $X$ is closed under arbitrary intersections, any subset $\Xi$ of $\mathcal{P}(T(X)) \times T(X)$ generates such a logic (which is then also said to be axiomatized by $\Xi)$. A pair $\Gamma / \varphi$ in $\mathcal{P}(T(X)) \times T(X)$ belongs to that logic iff $\varphi$ terminates some (possibly infinite) sequence, each item of which belongs to $\Gamma$ or is $h(\psi)$ for some endomorphism $h$ of $\boldsymbol{T}(X)$ and some pair $\Psi / \psi$ from $\Xi$, where $h[\Psi]$ consists of previous items of the sequence. (Here, $\Psi$ may be empty.) This observation goes back, in principle, to [30].

Definition 6.2. In a given algebraic signature, a logic over the proper class $\operatorname{Var}$ is a family $\vdash=\left\{\vdash^{X}: X \in \mathcal{P}(\operatorname{Var})\right\}$, where each $\vdash^{X}$ is a logic over $X$ (called a slice of $\vdash$ ) and the following variant of (iii) (called strong substitution-invariance) holds:

(iv) for any $X, Y \in \mathcal{P}(\operatorname{Var})$, if $\Gamma \vdash^{X} \varphi$, then $h[\Gamma] \vdash^{Y} h(\varphi)$ for all homomorphisms $h: \boldsymbol{T}(X) \rightarrow \boldsymbol{T}(Y)$.

In this case, the notation $\Gamma \vdash \varphi$ signifies that $\Gamma \vdash^{X} \varphi$ for some $X \in \mathcal{P}(\operatorname{Var})$, whence $\operatorname{var}(\Gamma \cup\{\varphi\}) \subseteq X$. (Conversely, if $\operatorname{var}(\Gamma \cup\{\varphi\}) \subseteq X \in \mathcal{P}(\operatorname{Var})$ and $\Gamma \vdash \varphi$, then $\Gamma \vdash^{X} \varphi$, by (iv).)

\footnotetext{
3 Terms in $T(X)$ correspond intuitively to assertions (as in Boolean algebra), but we resist the temptation to call them 'formulas', so as to prevent confusion with the first-order (e.g., atomic) formulas in the richer language of the class $\operatorname{Mod}^{*}(\vdash)$, defined below.
} 
Every logic $\vdash^{*}$ over a subset $X$ of the proper class $\operatorname{Var}$ may be viewed as the $X$-slice of a logic $\vdash$ over all of Var. One such $\vdash$, which we label as induced by $\vdash^{*}$, is defined by requiring that each of its slices $\vdash^{Y}$ be the logic over $Y$ generated by the set of all pairs $h[\Gamma] / h(\varphi)$ such that $\Gamma \vdash^{*} \varphi$ and $h: \boldsymbol{T}(X) \rightarrow \boldsymbol{T}(Y)$ is a homomorphism. (This $\vdash$ satisfies (iv), by the syntactic characterization of $\vdash^{Y}$ preceding Definition 6.2.)

Henceforth, $\vdash$ is assumed to be a logic either over Var or over an infinite subset of Var. Note that it is only for sets $\Gamma \cup\{\varphi\}$ that the notation $\Gamma \vdash \varphi$ is defined. All claims in the present section can be found in standard texts on abstract algebraic logic, e.g., [8, 14, 17] and the recent [16]. Their proofs are not affected by the extent of the class of variables. In fact, the class-versusset distinction will be unimportant, except in connection with Definitions 7.1 and 7.3 of Section 7.

If $\Gamma \vdash \varphi$, then the pair $\Gamma / \varphi$ is called a derivable rule of $\vdash$. The expression $\Gamma \vdash \Psi$ abbreviates ' $\Gamma \vdash \xi$ for all $\xi \in \Psi$ ', while $\Gamma \dashv \Psi$ means ' $\Gamma \vdash \Psi$ and $\Psi \vdash \Gamma$ ', and $\vdash \Psi$ stands for $\emptyset \vdash \Psi$. (The same conventions will apply to relations of the form $\models_{\mathrm{K}}$ below.)

A $(\vdash-)$ matrix $\langle\boldsymbol{A}, F\rangle$ comprises an algebra $\boldsymbol{A}$ in the signature of $\vdash$ and a set $F \subseteq A$. We regard it as a structure $\mathcal{A}$ for the signature whose operation symbols are the connectives of $\vdash$ and whose sole relation symbol $r$ is unary, so that $\mathcal{A} \models r(a)$ iff $a \in F$. Intuitively, $r$ is a 'truth' predicate. The substructures $\langle\boldsymbol{B}, B \cap F\rangle(\boldsymbol{B} \in \mathbb{S}(\boldsymbol{A}))$ of $\mathcal{A}$ are usually called submatrices. Similarly, matrix homomorphisms are the homomorphisms between matrices, considered as algebras with a distinguished unary relation.

Given a class $\mathrm{M}$ of matrices, we abbreviate $\{r(\gamma): \gamma \in \Gamma\} \models_{\mathrm{M}} r(\varphi)$ as $\Gamma \models_{M} \varphi$. When this is true, the rule $\Gamma / \varphi$ is said to be validated by (each member of) M. Abusing notation, we also use $\Gamma \models_{\mathrm{M}} \varphi \approx \psi$ to abbreviate $\{r(\gamma): \gamma \in \Gamma\} \models_{\mathrm{M}} \varphi \approx \psi$ (where $\Gamma$ still consists of terms, not equations).

A matrix $\langle\boldsymbol{A}, F\rangle$ is called a model of $\vdash$ if it validates all the derivable rules of $\vdash$, in which case $F$ is called a $\vdash-$ filter of $\boldsymbol{A}$. The set $F i_{\vdash} \boldsymbol{A}$ of all $\vdash$-filters of $\boldsymbol{A}$ is closed under arbitrary intersections and is therefore the universe of a complete lattice $\boldsymbol{F} \boldsymbol{i}_{\vdash} \boldsymbol{A}$, ordered by inclusion.

Given a matrix $\langle\boldsymbol{A}, F\rangle$, we denote by $\boldsymbol{\Omega}^{\boldsymbol{A}} F$ the largest congruence $\theta$ of $\boldsymbol{A}$ for which $F$ is a union of $\theta$-classes (i.e., for which $b \in F$ whenever both $\langle a, b\rangle \in \theta$ and $a \in F)$. This congruence always exists. If $h: B \rightarrow \boldsymbol{A}$ is a homomorphism and $F$ is a $\vdash$-filter of $\boldsymbol{A}$, then $h^{-1}[F]:=\{b \in B: h(b) \in F\}$ is a $\vdash$-filter of $\boldsymbol{B}$ and

$$
\boldsymbol{\Omega}^{\boldsymbol{B}} h^{-1}[F] \supseteq h^{-1}\left[\boldsymbol{\Omega}^{\boldsymbol{A}} F\right]:=\left\{\left\langle b, b^{\prime}\right\rangle \in B^{2}:\left\langle h(b), h\left(b^{\prime}\right)\right\rangle \in \boldsymbol{\Omega}^{\boldsymbol{A}} F\right\} .
$$

If, moreover, $h$ is surjective, then

$$
h^{-1}\left[\boldsymbol{\Omega}^{\boldsymbol{A}} F\right]=\boldsymbol{\Omega}^{\boldsymbol{B}} h^{-1}[F] .
$$

The maps $F \mapsto \boldsymbol{\Omega}^{\boldsymbol{A}} F\left(F \in F i_{\vdash} \boldsymbol{A}\right)$, taken over all algebras $\boldsymbol{A}$, constitute the Leibniz operator of $\vdash$. This operator is not always isotone, i.e., from $F, G \in F i_{\vdash} \boldsymbol{A}$ and $F \subseteq G$, it need not follow that $\boldsymbol{\Omega}^{\boldsymbol{A}} F \subseteq \boldsymbol{\Omega}^{\boldsymbol{A}} G$. 
A matrix $\langle\boldsymbol{A}, F\rangle$ is said to be reduced if $\boldsymbol{\Omega}^{\boldsymbol{A}} F=\operatorname{id}_{A}:=\{\langle a, a\rangle: a \in A\}$. The derivable rules of $\vdash$ are exactly the pairs $\Gamma / \varphi$ validated by the class $\operatorname{Mod}^{*}(\vdash)$ of all reduced matrix models of $\vdash$ (i.e., $\Gamma \vdash \varphi$ iff $\left.\Gamma \models \operatorname{Mod}^{*}(\vdash) \varphi\right)$. We treat $\operatorname{Mod}^{*}(\vdash)$ as a concrete category, equipped with all matrix homomorphisms between its members.

Theorem 6.3. The following conditions on $\vdash$ are equivalent.

(i) $\operatorname{Mod}^{*}(\vdash)$ is a prevariety.

(ii) The Leibniz operator of $\vdash$ is isotone (for all algebras) and (3) holds for all homomorphisms $h: \boldsymbol{B} \rightarrow \boldsymbol{A}$ and all $\vdash$-filters $F$ of $\boldsymbol{A}$.

(iii) There exists a set $\Delta$ of binary terms such that, for any matrix model $\langle\boldsymbol{A}, F\rangle$ of $\vdash$, we have $\boldsymbol{\Omega}^{\boldsymbol{A}} F=\left\{\langle a, b\rangle \in A^{2}: \Delta^{\boldsymbol{A}}(a, b) \subseteq F\right\}$.

(iv) There exists a set $\Delta$ of binary terms such that

$$
\begin{aligned}
& \vdash \Delta(x, x) ; \\
& \{x\} \cup \Delta(x, y) \vdash y ; \\
& \Delta\left(x_{1}, y_{1}\right) \cup \ldots \cup \Delta\left(x_{n}, y_{n}\right) \vdash \Delta\left(\varphi\left(x_{1}, \ldots, x_{n}\right), \varphi\left(y_{1}, \ldots, y_{n}\right)\right),
\end{aligned}
$$

for every connective $\varphi$ of $\vdash$, where $n$ is the rank of $\varphi$.

A set $\Delta$ of binary terms witnesses (iii) iff it witnesses (iv). In that case, the third demand in (iv) generalizes from connectives $\varphi$ to arbitrary terms.

We say that $\vdash$ is equivalential if the conditions in Theorem 6.3 hold. The elements of the set $\Delta$ in (iii) or (iv) are then called equivalence formulas for $\vdash$, and they are unique in the sense that $\Delta(x, y) \dashv \vdash \Delta^{\prime}(x, y)$ for any other such set $\Delta^{\prime}$. In this case, $\Delta(x, y) \models_{\operatorname{Mod}^{*}(\vdash)} x \approx y$, by (iii). For the roots of Theorem 6.3, see [38, pp. 222-3], as well as [9, 13, 14, 23, 34].

Remark 6.4. Suppose $\Delta$ is a set of equivalence formulas for $\vdash$, with $r$ as above. Then $\operatorname{Mod}^{*}(\vdash)$ is clearly axiomatized by the implications

$$
\{r(\gamma): \gamma \in \Gamma\} / r(\varphi)
$$

corresponding to the rules $\Gamma / \varphi$ in any given axiomatization $\Xi$ of $\vdash$, together with the postulate

$$
\{r(\rho(x, y)): \rho \in \Delta\} / x \approx y .
$$

Thus, if $\operatorname{var}(\Xi)$ is a set, then $\operatorname{Mod}^{*}(\vdash)$ is a $\left(|\operatorname{var}(\Xi)|+\aleph_{0}\right)$-prevariety. In general, $\operatorname{Mod}^{*}(\vdash)$ is a quasivariety iff $\vdash$ is finitely equivalential (i.e., equipped with a finite set of equivalence formulas) and finitary [13, 9].

We define $\operatorname{Alg}^{*}(\vdash)=\left\{\boldsymbol{A}:\langle\boldsymbol{A}, F\rangle \in \operatorname{Mod}^{*}(\vdash)\right.$ for some $\left.F\right\}$.

Given a class $\mathrm{K} \cup\{\boldsymbol{A}\}$ of similar algebras, let $\operatorname{Con}_{\mathrm{K}} \boldsymbol{A}$ denote the set of all $\mathrm{K}$-congruences of $\boldsymbol{A}$, i.e., all congruences $\theta$ such that $\boldsymbol{A} / \theta \in \mathrm{K}$. If $\mathrm{K}$ is a prevariety, then $\operatorname{Con}_{\mathrm{K}} \boldsymbol{A}$ is closed under arbitrary intersections and is therefore the universe of a complete lattice, $\boldsymbol{C o n}_{\mathrm{K}} \boldsymbol{A}$, ordered by inclusion. 
Theorem 6.5. The following conditions on $\vdash$ are equivalent.

(i) $\vdash$ is equivalential and its reduced matrix models are determined by their algebra reducts, i.e., whenever $\langle\boldsymbol{A}, F\rangle,\langle\boldsymbol{A}, G\rangle \in \operatorname{Mod}^{*}(\vdash)$, then $F=G$.

(ii) Alg* $(\vdash)$ is a prevariety and, for each algebra $\boldsymbol{A}$, the map $F \mapsto \boldsymbol{\Omega}^{\boldsymbol{A}} F$ defines a lattice isomorphism from $\boldsymbol{F i}_{\vdash} \boldsymbol{A}$ onto $\boldsymbol{C o n}_{\mathrm{Alg}^{*}(\vdash)} \boldsymbol{A}$.

(iii) There exist a class $\mathrm{K}$ of algebras, a set $\left\{\left\langle\delta_{i}, \varepsilon_{i}\right\rangle: i \in I\right\}$ of pairs of unary terms and a set $\Delta$ of binary terms such that, for any set $\Gamma \cup\{\varphi\}$ of terms,

$$
\begin{gathered}
\Gamma \vdash \varphi \text { iff }\left\{\delta_{i}(\gamma) \approx \varepsilon_{i}(\gamma): \gamma \in \Gamma, i \in I\right\} \models_{\mathrm{K}}\left\{\delta_{i}(\varphi) \approx \varepsilon_{i}(\varphi): i \in I\right\} ; \\
\left\{\delta_{i}(\rho(x, y)) \approx \varepsilon_{i}(\rho(x, y)): i \in I, \rho \in \Delta\right\}=\models_{\mathrm{K}} x \approx y .
\end{gathered}
$$

In this case, $\Delta$ is a set of equivalence formulas for $\vdash$, and $\operatorname{Alg}^{*}(\vdash)$ is the unique prevariety $\mathrm{K}$ of algebras for which (iii) holds.

We say that $\vdash$ is algebraizable and, more explicitly, that $\operatorname{Alg}^{*}(\vdash)$ algebraizes $\vdash$, if the conditions of Theorem 6.5 hold. The pairs in (iii) are then unique in the sense that

$$
\left\{\delta_{i}(x) \approx \varepsilon_{i}(x): i \in I\right\}==_{\mathrm{Alg}^{*}(\vdash)}\left\{\delta_{j}^{\prime}(x) \approx \varepsilon_{j}^{\prime}(x): j \in J\right\}
$$

for any other such set $\left\{\left\langle\delta_{j}^{\prime}, \varepsilon_{j}^{\prime}\right\rangle: j \in J\right\}$. In this case, when $\langle\boldsymbol{A}, F\rangle \in \operatorname{Mod}^{*}(\vdash)$, then $F=\left\{a \in A: \delta_{i}^{\boldsymbol{A}}(a)=\varepsilon_{i}^{\boldsymbol{A}}(a)\right.$ for all $\left.i \in I\right\}$. The concrete categories $\operatorname{Mod}^{*}(\vdash)$ and $\operatorname{Alg}^{*}(\vdash)$ are therefore isomorphic when $\vdash$ is algebraizable.

The original definition of algebraizability is due to Blok and Pigozzi [8]. Its scope was widened in [7, 14, 23] and adapted to logics over proper classes in [15]. For the origins of Theorem 6.5, see [8, 9] also.

Remark 6.6. When the conditions of Theorem 6.5 hold, then $\vdash$ is axiomatized by the postulates captured in Theorem 6.3(iv), in the relation

$$
x \dashv \vdash \bigcup_{i \in I} \Delta\left(\delta_{i}(x), \varepsilon_{i}(x)\right)
$$

and in the rules $\bigcup_{\langle\xi, \eta\rangle \in \Sigma} \Delta(\xi, \eta) \vdash \Delta(\varphi, \psi)$ corresponding to the equational implications $\Sigma / \varphi \approx \psi$ belonging to any axiomatization of $\operatorname{Alg}^{*}(\vdash)$. In this case, therefore, if $\operatorname{Alg}^{*}(\vdash)$ is an $\mathfrak{m}$-prevariety, then $\vdash$ can be axiomatized using at most $\mathfrak{m}$ variables.

\section{Beth Definability Properties}

Definition 7.1. ([6]) A logic $\vdash$ over the proper class Var is said to have the (deductive) infinite Beth (definability) property if the following holds for all disjoint subsets $X, Z$ of $\operatorname{Var}$, with $T(X) \neq \emptyset$, and all $\Gamma \subseteq T(X \cup Z)$ : if,

for each $z \in Z$ and each homomorphism $h: \boldsymbol{T}(X \cup Z) \rightarrow \boldsymbol{T}(Y)$, with $Y \in \mathcal{P}(\operatorname{Var})$, such that $h(x)=x$ for all $x \in X$, we have

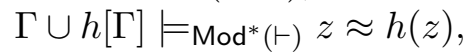

then, for each $z \in Z$, there exists $\varphi_{z} \in T(X)$ such that $\Gamma \models_{\operatorname{Mod}^{*}(\vdash)} z \approx \varphi_{z}$. 
Theorem 7.2. ([6, Thm. 3.12]) Let $\vdash$ be an equivalential logic over a proper class. Then $\vdash$ has the infinite Beth property iff, in the prevariety $\operatorname{Mod}^{*}(\vdash)$, all epimorphisms are surjective.

We have not found the following definition in the published literature.

Definition 7.3. Let $\vdash$ be a logic over an infinite set $V$. We shall say that $\vdash$ has the $(V-)$ localized infinite Beth property provided that the following is true for all disjoint subsets $X, Z$ of $V$ and all $\Gamma \subseteq T(X \cup Z)$, such that $T(X) \neq \emptyset$ and $|V \backslash(X \cup Z)| \geq|Z|+\aleph_{0}$ : if,

for each $z \in Z$ and each endomorphism $h$ of $\boldsymbol{T}(V)$, such that

$h(x)=x$ for all $x \in X$, we have $\Gamma \cup h[\Gamma] \models \operatorname{Mod}^{*}(\vdash) z \approx h(z)$,

then, for each $z \in Z$, there exists $\varphi_{z} \in T(X)$ such that $\Gamma \models_{\operatorname{Mod}^{*}(\vdash)} z \approx \varphi_{z}$.

The displayed assumptions in Definitions 7.1 and 7.3 will both be pronounced as ' $\Gamma$ defines $Z$ implicitly in terms of $X$ in $\vdash$ '. (There is no ambiguity, since the two possibilities for $\vdash$ are mutually exclusive.) The term $\varphi_{z}$ in the conclusion is called an explicit definition of $z$ in terms of $X$, with respect to $\Gamma$, in $\vdash$.

If $\Delta$ is a set of equivalence formulas for $\vdash$, then in Definitions 7.1 and

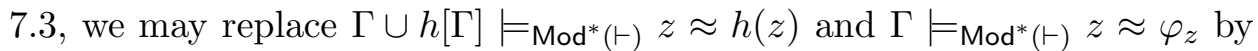
the intrinsic (but equivalent) respective demands

$$
\Gamma \cup h[\Gamma] \vdash \Delta(z, h(z)) \text { and } \Gamma \vdash \Delta\left(z, \varphi_{z}\right) \text {. }
$$

Lemma 7.4. Let $\vdash$ be an equivalential logic over the proper class Var, and let $V \in \mathcal{P}($ Var $)$ be infinite. If $\vdash$ has the infinite Beth property, then $\vdash^{V}$ has the localized infinite Beth property.

Proof. It is given that $\vdash$ has a set $\Delta$ of equivalence formulas. Let $X, Z$ and $\Gamma$ be as in Definition 7.3, and $h: \boldsymbol{T}(X \cup Z) \rightarrow \boldsymbol{T}(X \cup \bar{Z})$ as in Definition 7.1, where $\bar{Z}:=\operatorname{var}(h[Z])$. Set $W=V \backslash(X \cup Z)$. As $V$ is infinite and $\Delta$ consists of binary terms, we may assume that $\Delta \subseteq T(V)$. In view of Theorem 6.3 (iv), $\Delta$ is also a set of equivalence formulas for $\vdash^{V}$, so we need only show that $\Gamma \cup h[\Gamma] \vdash \Delta(z, h(z))$ for all $z \in Z$.

As $|\bar{Z}| \leq|Z|+\aleph_{0} \leq|W|$, there are homomorphisms $f: \boldsymbol{T}(\bar{Z}) \rightarrow \boldsymbol{T}(W)$ and $g: \boldsymbol{T}(W) \rightarrow \boldsymbol{T}(\bar{Z})$, with $g \circ f=\operatorname{id}_{T(\bar{Z})}$. Let $q$ be the endomorphism of $\boldsymbol{T}(V)$ that agrees with $f \circ h$ on $Z$ and that fixes all elements of $V \backslash Z$. Assuming that $\Gamma$ defines $Z$ implicitly in terms of $X$ in $\vdash^{V}$, we infer that $\Gamma \cup q[\Gamma] \vdash^{V} \Delta(z, q(z))$ for all $z \in Z$. Then, by Definition 6.2(iv),

$$
p[\Gamma] \cup p q[\Gamma] \vdash p[\Delta(z, q(z))] \text { for all } z \in Z,
$$

where $p: \boldsymbol{T}(V) \rightarrow \boldsymbol{T}(X \cup Z \cup \bar{Z})$ is the homomorphism that agrees with $g$ on $W$, while fixing all elements of $X \cup Z$. Now (4) simplifies to

$$
\Gamma \cup h[\Gamma] \vdash \Delta(z, h(z)) \text { for all } z \in Z,
$$

by the definitions of $p$ and $q$, and since $g \circ f=\operatorname{id}_{T(\bar{Z})}$. 
Theorem 7.5. Let $\vdash$ be an equivalential logic over an infinite set $V$, where $\vdash$ has at most $|V|$ connectives and has the localized infinite Beth property. Then no member of $\operatorname{Mod}^{*}(\vdash)$ with at most $|V|$ elements has a proper $\operatorname{Mod}^{*}(\vdash)$-epic submatrix.

Proof. As $V$ is infinite, it can be partitioned into sets $V^{\prime}$ and $V \backslash V^{\prime}$, both of cardinality $|V|$. Let $\mathcal{B}=\left\langle\boldsymbol{B}, F_{B}\right\rangle \in \operatorname{Mod}^{*}(\vdash)$, with $|B| \leq|V|$, and consider a $\operatorname{Mod}^{*}(\vdash)$-epic submatrix $\mathcal{A}=\left\langle\boldsymbol{A}, A \cap F_{B}\right\rangle$ of $\mathcal{B}$. As $|B| \leq\left|V^{\prime}\right|$, there is a surjection $V^{\prime} \rightarrow B$, which may be extended to a homomorphism $h: \boldsymbol{T}\left(V^{\prime}\right) \rightarrow \boldsymbol{B}$. Assume, with a view to contradiction, that $A \neq B$, and let

$$
X=V^{\prime} \cap h^{-1}[A] \text { and } Z=V^{\prime} \cap h^{-1}[B \backslash A],
$$

so $X \cup Z=V^{\prime}$ and $X \cap Z=\emptyset \neq T(X)$. Then $\Gamma:=h^{-1}\left[F_{B}\right] \in F i_{\vdash} \boldsymbol{T}\left(V^{\prime}\right)$.

As $V \backslash(X \cup Z)=V \backslash V^{\prime}$ has the same (infinite) cardinality as $V^{\prime}$, which contains $Z$, we have $|V \backslash(X \cup Z)| \geq|Z|+\aleph_{0}$. Therefore, because $\mathcal{A}$ is $\operatorname{Mod}^{*}(\vdash)$-epic in $\mathcal{B}$, it can be shown, just as in the proof of [6, Thm. 3.12], that for any endomorphism $k$ of $\boldsymbol{T}(V)$ which fixes all elements of $X$, and any homomorphism $g: \boldsymbol{T}(V) \rightarrow \boldsymbol{C}$, where $\left\langle\boldsymbol{C}, F_{C}\right\rangle \in \operatorname{Mod}^{*}(\vdash)$,

$$
\text { if } g[\Gamma \cup k[\Gamma]] \subseteq F_{C} \text {, then } g(z)=g(k(z)) \text { for all } z \in Z \text {. }
$$

That is, $\Gamma$ defines $Z$ implicitly in terms of $X$ in $\vdash$.

Pick $b \in B \backslash A$. As $\left.h\right|_{V^{\prime}}: V^{\prime} \rightarrow B$ is surjective, we have $b=h(z)$ for some $z \in Z$. By the localized infinite Beth property, $\Gamma \models_{\operatorname{Mod}^{*}(\vdash)} z \approx \varphi_{z}$ for some $\varphi_{z}=\varphi_{z}(\vec{x}) \in T(X)$. As $\mathcal{B} \in \operatorname{Mod}^{*}(\vdash)$ and $h[\Gamma] \subseteq F_{B}$, it follows that

$$
b=h(z)=h\left(\varphi_{z}\right)=\varphi^{B}(h[\vec{x}]) .
$$

Now $h[\vec{x}]$ consists of elements of $A$, and $\boldsymbol{A} \in \mathbb{S}(\boldsymbol{B})$, so $b \in A$. This contradiction shows that $A=B$, as required.

We can now prove the following bridge theorem, in which the need to consider proper classes is eliminated for symbolically limited logics.

Theorem 7.6. Let $\vdash$ be an equivalential logic over an infinite subset $V$ of the proper class Var. Let $\mathfrak{s}$ be the cardinality of the signature of $\vdash$, and assume that $\vdash$ has an axiomatization that uses at most $\mathfrak{m}$ variables, where $\mathfrak{m}+\mathfrak{s} \leq|V|$. Then the following conditions are equivalent.

(i) $\vdash$ has the localized infinite Beth property with respect to $V$.

(ii) No member of $\operatorname{Mod}^{*}(\vdash)$ with at most $|V|$ elements has a proper $\operatorname{Mod}^{*}(\vdash)$-epic submatrix.

(iii) All epimorphisms in $\operatorname{Mod}^{*}(\vdash)$ are surjective.

(iv) The logic over Var induced by $\vdash$ has the infinite Beth property.

Proof. (ii) $\Rightarrow$ (iii) instantiates Theorem 7.5 .

(iii) $\Rightarrow$ (iii): By Remark 6.4. $\operatorname{Mod}^{*}(\vdash)$ is a $|V|$-prevariety, so the present implication follows from Theorem 5.1 . 
(iii) $\Rightarrow$ (iv): As $\vdash$ and the induced logic $\vdash^{\prime}$ have the same matrix models, $\operatorname{Mod}^{*}(\vdash)=\operatorname{Mod}^{*}\left(\vdash^{\prime}\right)$. The implication therefore follows from Theorem 7.2 , (iv) $\Rightarrow$ (ii) instantiates Lemma 7.4.

Corollary 7.7. Theorem $[7.6$ remains true for algebraizable logics $\vdash$ if we replace the terms 'submatrix' and 'Mod* $(\vdash)$ ' by 'subalgebra' and 'Alg*( $\vdash)$ ', respectively.

Proof. Apply to Theorem 7.6 the category isomorphism between $\operatorname{Mod}^{*}(\vdash)$ and $\operatorname{Alg}^{*}(\vdash)$ that is guaranteed by the algebraizability of $\vdash$.

Corollary 7.8. Let $\vdash$ be a logic with a countable signature, over a denumerable set $V$ of variables. Consider the following conditions.

(i) $\vdash$ has the localized infinite Beth property with respect to $V$.

(ii) $\operatorname{Mod}^{*}(\vdash)$ has the ES property.

(iii) $\operatorname{Alg}^{*}(\vdash)$ has the ES property.

If $\vdash$ is finitary and equivalential, then (ii) and (iii) are equivalent.

If $\vdash$ is algebraized by a quasivariety, then (ii) and (iii) are equivalent.

Proof. A finitary equivalential logic with a countable signature satisfies the initial hypotheses of Theorem 7.6 when $\mathfrak{m}=\aleph_{0}$. For the second claim, use Remark 6.6 (with $\left.\mathfrak{m}=\aleph_{0}\right)$ and Corollary 7.7.

Even for algebraizable logics, the finiteness assumptions in the two claims of Corollary 17.8 are mutually independent: see [22] and [36], respectively.

The finite Beth property is defined like the infinite one, except that the set $Z$ in its definition is required to be finite. An equivalential logic $\vdash$ over a proper class has this property iff $\operatorname{Mod}^{*}(\vdash)$ has the weak ES property [6, Thm. 3.14, Cor. 3.15].

Let us also define the $(V-)$ localized finite Beth property like its infinite analogue, but stipulating that $Z$ be finite and substituting ' $V \backslash X$ is infinite' for ' $|V \backslash(X \cup Z)| \geq|Z|+\aleph_{0}$ '.

Theorem 7.6 and its corollaries have analogues for these properties. We state only one, wherein the cardinality of the signature plays no role.

Theorem 7.9. Let $\vdash$ be a logic over a denumerable subset $V$ of the proper class Var. Consider the following conditions.

(i) $\vdash$ has the localized finite Beth property with respect to $V$.

(ii) No finitely generated member of $\operatorname{Mod}^{*}(\vdash)$ has a proper $\operatorname{Mod}^{*}(\vdash)-$ epic submatrix.

(iii) $\operatorname{Mod}^{*}(\vdash)$ has the weak ES property.

(iv) The logic over Var induced by $\vdash$ has the finite Beth property.

(v) No finitely generated member of $\operatorname{Alg}^{*}(\vdash)$ has a proper $\operatorname{Alg}^{*}(\vdash)$-epic subalgebra.

(vi) $\operatorname{Alg}^{*}(\vdash)$ has the weak ES property. 
If $\vdash$ is finitary and finitely equivalential, then (ii)-(iv) are equivalent.

If $\vdash$ is algebraized by a quasivariety, then (ii), (iv), (v) and (vi) are equivalent (even if $\vdash$ is not finitary).

Proof. (ii) $\Rightarrow$ (iii): Adapt the proof of Theorem [7.5, arranging that $h$ maps $V^{\prime}$ onto a finite generating set for $\boldsymbol{B}$. At the end, instead of $b=h(z)$, we have $b=\psi^{\boldsymbol{B}}(h[\vec{x}], h[\vec{z}])$ for a suitable term $\psi$ and finite sequences $\vec{x} \in X$ and $\vec{z} \in Z$. Apply the original argument to the items in $\vec{z}$.

Both (iii) $\Rightarrow$ (iii) and (V) $\Rightarrow$ (vi) instantiate Theorem [5.4, because $\operatorname{Mod}^{*}(\vdash)$ is a quasivariety even in the former case (see Remark 6.4).

Otherwise, the proof is like that of Theorem 7.6 and its corollaries.

For equivalential logics, the meaning of the finite Beth property is not affected if we stipulate in its definition that $Z$ be a singleton. This is deduced from relevant bridge theorems in [6, Cor. 3.15], and the argument applies equally to the localized finite Beth property. Therefore, by the proof in [29], all six conditions of Theorem 7.9 hold when $\vdash$ is an axiomatic extension of intuitionistic propositional logic, or of its positive fragment (cf. Remark 5.3).

For simplicity, we have confined the above discussion to sentential logics, but the results of this section extend straightforwardly to the ' $k$-deductive systems' of [6, 9] and to Gentzen systems as formulated, for instance, in [35].

\section{REFERENCES}

[1] J. Adámek, How many variables does a quasivariety need? Algebra Universalis 27 (1990), 44-48.

[2] P.D. Bacsich, Model theory of epimorphisms, Canad. Math. Bull. 17 (1974), 471-477.

[3] B. Banaschewski, H. Herrlich, Subcategories defined by implications, Houston J. Math. 2 (1976), 149-171.

[4] G. Bezhanishvili, T. Moraschini, J.G. Raftery, Epimorphisms in varieties of residuated structures, J. Algebra 492 (2017), 185-211.

[5] G. Birkhoff, On the structure of abstract algebras, Proc. Cambridge Phil. Soc. 29 (1935), 433-454.

[6] W.J. Blok, E. Hoogland, The Beth property in algebraic logic, Studia Logica 83 (2006), 49-90.

[7] W.J. Blok, B. Jónsson, Equivalence of consequence operations, Studia Logica $\mathbf{8 3}$ (2006), 91-110.

[8] W.J. Blok, D. Pigozzi, 'Algebraizable Logics', Memoirs of the American Mathematical Society 396, Amer. Math. Soc., Providence, 1989.

[9] W.J. Blok, D. Pigozzi, Algebraic semantics for universal Horn logic without equality, in J.D.H. Smith, A. Romanowska (eds.), 'Universal Algebra and Quasigroup Theory', Heldermann Verlag, Berlin, 1992, pp. 1-56.

[10] A. Budkin, Dominions in quasivarieties of universal algebras, Studia Logica $\mathbf{7 8}$ (2004), 107-127.

[11] A. Budkin, Dominions of universal algebras and projective properties, Algebra and Logic 47 (2008), 304-313.

[12] M.A. Campercholi, Dominions and primitive positive functions, J. Symbolic Logic 83 (2018), 40-54.

[13] J. Czelakowski, Equivalential logics (I), and (II), Studia Logica 40 (1981), 227-236, and 355-372. 
[14] J. Czelakowski, 'Protoalgebraic Logics', Kluwer, Dordrecht, 2001.

[15] J. Czelakowski, D. Pigozzi, Amalgamation and interpolation in abstract algebraic logic, in X. Caicedo, C.H. Montenegro (eds.), 'Models, Algebras and Proofs', Lecture Notes in Pure and Applied Mathematics, No. 203, Marcel Dekker, New York, 1999, pp. 187-265.

[16] J.M. Font, 'Abstract Algebraic Logic - An Introductory Textbook', Studies in Logic 60, College Publications, London, 2016.

[17] J.M. Font, R. Jansana, D. Pigozzi, A survey of abstract algebraic logic, and Update, Studia Logica 74 (2003), 13-97, and 91 (2009), 125-130.

[18] P. Freyd, 'Abelian categories', Harper and Row, New York, 1964.

[19] D.M. Gabbay, L. Maksimova, 'Interpolation and Definability: Modal and Intuitionistic Logics', Oxford Logic Guides 46, Clarendon Press, Oxford, 2005.

[20] V.A. Gorbunov, 'Algebraic Theory of Quasivarieties', Consultants Bureau, New York, 1998.

[21] G. Grätzer, H. Lakser, A note on the implicational class generated by a class of structures, Canad. Math. Bull. 16 (1973), 603-605.

[22] B. Herrmann, Equivalential and algebraizable logics, Studia Logica 57 (1996), 419436.

[23] B. Herrmann, Characterizing equivalential and algebraizable logics by the Leibniz operator, Studia Logica 58 (1997), 305-323.

[24] L. Henkin, J.D. Monk, A. Tarski, 'Cylindric Algebras, Part II', North-Holland, Amsterdam, 1985.

[25] P. Higgins, Epimorphisms and amalgams, Colloquium Mathematicum 56 (1988), $1-17$.

[26] E. Hoogland, Algebraic characterizations of various Beth definability properties, Studia Logica 65 (2000), 91-112.

[27] E. Hoogland, 'Definability and interpolation: model-theoretic investigations', PhD. Thesis, Institute for Logic, Language and Computation, University of Amsterdam, 2001.

[28] J.R. Isbell, Epimorphisms and dominions, in S. Eilenberg et al (eds.), 'Proceedings of the Conference on Categorical Algebra (La Jolla, California, 1965)', Springer, New York, 1966, pp. 232-246.

[29] G. Kreisel, Explicit definability in intuitionistic logic, J. Symbolic Logic 25 (1960), 389-390.

[30] J. Łoś, R. Suszko, Remarks on sentential logics, Proc. Kon. Nederl. Akad. van Wetenschappen, Series A 61 (1958), 177-183.

[31] L.L. Maksimova, Intuitionistic logic and implicit definability, Ann. Pure Appl. Logic 105 (2000), 83-102.

[32] L.L. Maksimova, Implicit definability and positive logics, Algebra and Logic 42 (2003), 37-53.

[33] A.I. Maltsev, Several remarks on quasivarieties of algebraic systems (Russian), Algebra i Logika 5 (1966), 3-9.

[34] T. Prucnal, A. Wroński, An algebraic characterization of the notion of structural completeness, Bull. Sect. Logic 3 (1974), 30-33.

[35] J.G. Raftery, Correspondences between Gentzen and Hilbert systems, J. Symbolic Logic 71 (2006), 903-957.

[36] J.G. Raftery, A non-finitary sentential logic that is elementarily algebraizable, J. Logic Comput. 20 (2010), 969-975.

[37] D. Wasserman, 'Epimorphisms and Dominions in Varieties of Lattices', PhD thesis, University of California at Berkeley, 2001.

[38] R. Wójcicki, 'Theory of Logical Calculi', Kluwer, Dordrecht, 1988. 
Institute of Computer Science, Academy of Sciences of the Czech Republic, Pod Vodárenskou věží 2, 18207 Prague 8, Czech Republic.

E-mail address: moraschini@cs.cas.cz

Department of Mathematics and Applied Mathematics, University of Pretoria, Private Bag X20, Hatfield, Pretoria 0028, South Africa

E-mail address: james.raftery@up.ac.za

Department of Mathematics and Applied Mathematics, University of Pretoria, Private Bag X20, Hatfield, Pretoria 0028, and DST-NRF Centre of ExCellence in Mathematical and Statistical Sciences (CoE-MaSS), South Africa

E-mail address: jamie.wannenburg@up.ac.za 